\title{
Limb immobilization alters functional electrophysiological parameters of sciatic nerve
}

\author{
J.S.M. Alves ${ }^{1}$, J.H. Leal-Cardoso ${ }^{1}$, F.F.U. Santos-Júnior ${ }^{1}$, P.S. Carlos ${ }^{1}$, R.C. Silva ${ }^{3}$, C.M. Lucci ${ }^{3}$, \\ S.N. Báo ${ }^{3}$, V.M. Ceccatto ${ }^{1}$ and R. Barbosa ${ }^{2}$ \\ ${ }^{1}$ Instituto Superior de Ciências Biomédicas, Universidade Estadual do Ceará, Fortaleza, CE, Brasil \\ ${ }^{2}$ Universidade Regional do Cariri, Crato, CE, Brasil \\ ${ }^{3}$ Instituto de Ciências Biológicas, Universidade de Brasília, Brasília, DF, Brasil
}

\begin{abstract}
Immobilization, used in clinical practice to treat traumatologic problems, causes changes in muscle, but it is not known whether changes also occur in nerves. We investigated the effects of immobilization on excitability and compound action potential (CAP) and the ultrastructure of the rat sciatic nerve. Fourteen days after immobilization of the right leg of adult male Wistar rats $(n=34)$, animals were killed and the right sciatic nerve was dissected and mounted in a moist chamber. Nerves were stimulated at a baseline frequency of $0.2 \mathrm{~Hz}$ and tested for $2 \mathrm{~min}$ at 20,50, and $100 \mathrm{~Hz}$. Immobilization altered nerve excitability. Rheobase and chronaxy changed from $3.13 \pm 0.05 \mathrm{~V}$ and $52.31 \pm 1.95 \mu \mathrm{s}$ (control group, $\mathrm{n}=13$ ) to $2.84 \pm 0.06 \mathrm{~V}$ and $59.71 \pm 2.79 \mu \mathrm{s}$ (immobilized group, $\mathrm{n}=15$ ), respectively. Immobilization altered the amplitude of CAP waves and decreased the conduction velocity of the first CAP wave (from $93.63 \pm 7.49$ to $79.14 \pm 5.59 \mathrm{~m} / \mathrm{s}$ ) but not of the second wave. Transmission electron microscopy showed fragmentation of the myelin sheath of the sciatic nerve of immobilized limbs and degeneration of the axon. In conclusion, we demonstrated that long-lasting leg immobilization can induce alterations in nerve function.
\end{abstract}

Key words: Immobilization; Sciatic nerve; Compound action potential; Conduction velocity; Rheobase; Chronaxy

\section{Introduction}

Peripheral nerves are frequent targets of traumatic injuries, such as crushing, compression, stretching, avulsion, and partial or full section, resulting in impaired nerve impulse transmission and reduction or loss of sensation and motor function in the innervated area. The magnitude of the changes in nerve function depends upon the nature of the injury, timing, type and diameter of the affected nerve fibers $(1,2)$. Traumatic injuries lead to partial or total limb disability that prevents the patient from performing their usual activities for long periods of time (3). As a part of the healing process, neuromuscular plasticity develops. As an important component of the neural basis of control of movement, plasticity also occurs as a result of alterations in the use of neuromuscular body components within the normal range of function (3).

Traumatic injuries and their treatments frequently lead to long-lasting limb immobilization. Since the control of movement and associated neuromuscular plasticity depends on the continued response to the nervous system, neuromuscular plasticity is expected to occur with simple immobilization. Indeed, it is already documented that long-lasting body immobilization causes skeletal muscle atrophy $(4,5)$ and it would be predicted that alterations of nerve function are likely to occur. It is also known that innervation is critical for the functional and structural integrity of muscle $(6,7)$, with degeneration of nerve function causing loss of muscle weight and the ability to generate muscular force $(8,9)$. Since simple immobilization may cause negative plasticity in muscle, it is important to determine whether functional alterations also occur in nerves as a result of simple immobilization. If they do occur, it is important to learn the underlying mechanism in order to understand the contribution of negative plasticity due to immobilization during the healing of traumatic injuries on nerve tissue.

Few published studies have assessed immobilizationinduced alteration of physiological and morphological parameters of the sciatic nerve (SN). Since long-lasting

Correspondence: J.H. Leal-Cardoso, Instituto Superior de Ciências Biomédicas, Universidade Estadual do Ceará, Av. Paranjana, 1700, 60740-000 Fortaleza, CE, Brasil. E-mail: lealcard@yahoo.com 
limb immobilization is frequent in clinical practice, the objective of the present study was to determine whether immobilization induces alterations of electrophysiological parameters related to excitability of peripheral nerves and, if so, to elucidate the mechanism. In the present study, we examined whether immobilization of the right hind paw of the rat for 14 days would alter electrophysiological and morphological properties of the SN. We found that this experimental condition altered the excitability of the nerve as demonstrated by modifications of rheobase, chronaxy, and various parameters of the compound nerve action potential (CAP). Immobilization also induced axonal and myelin sheath degeneration.

\section{Material and Methods}

\section{Dissection, tissue preparation, and immobilization protocol}

Male Wistar rats (Rattus norvegicus, 180-200 g, $\mathrm{n}=34$ ) were used. All animals were maintained in compliance with the Guide for Care and Use of Laboratory Animals published by the U.S. National Institutes of Health (NIH publication 27-89, revised 1996; http://www.nap.edu) to minimize suffering. All procedures described here were evaluated by, and received the approval of the Ethics Committee for Animal Use at the Universidade Estadual do Ceará (project No. 08351783-9).

Immobilization was performed by a method similar to that described by Santos-Júnior et al. (10) in 8-week-old animals. Briefly, the rats were first anesthetized with $60 \mathrm{mg} / \mathrm{kg}$ ketamine and $8 \mathrm{mg} / \mathrm{kg}$ xylazine, intraperitoneally. A control group received no immobilization. In the experimental group, waterproof tape was wrapped around the pelvis, hip, knee, and ankle of the right hind leg in order to achieve full immobilization.

At the end of treatment (14 days of immobilization), the rats were killed by cerebral concussion and the right $\mathrm{SN}$ was dissected. The tissues were then immediately placed in Petri dishes containing refrigerated Locke's solution. Nerves were mounted in a moist chamber and used on the day of dissection for recording of CAP. Petri dishes and moist chambers contained modified Locke's solution, $\mathrm{pH} 7.4$, containing $140 \mathrm{mM} \mathrm{NaCl}, 5.6 \mathrm{mM} \mathrm{KCl}$, $1.2 \mathrm{mM} \mathrm{MgCl}_{2}, 2.2 \mathrm{mM} \mathrm{CaCl}_{2}, 10 \mathrm{mM}$ Tris (hydroxymethylaminomethane), and $10 \mathrm{mM}$ glucose. The experiments were performed at room temperature $\left(18-22^{\circ} \mathrm{C}\right)$. All salts were purchased from Sigma Chemical (USA) or Reagen (Brazil) and were of analytical grade.

\section{Electrophysiological recording}

The SN was stimulated, and evoked CAP was recorded as described by Lima-Accioly et al. (11). Briefly, the SN was mounted in a moist chamber and one end was stimulated with electrical pulses at a baseline frequency of $0.2 \mathrm{~Hz}$. A $100 \mu \mathrm{s}, 40 \mathrm{~V}$ pulse was delivered to the $\mathrm{SN}$ by a stimulus isolation unit (Model
SIU4678, Grass Instruments Co., USA) connected to a stimulator (Model S48, Grass Instruments Co.). Evoked CAPs were recorded with platinum electrodes placed 4-5 $\mathrm{cm}$ from the stimulation electrodes. The recording electrodes were connected to an oscilloscope (Model 547, Tektronix, Inc., USA) through a high input impedance amplifier (model AM 01/UECE) specially configured and produced in the laboratory to meet the needs of the research. Digidata 1200 acquisition, computer hardware (Axon Instruments, Inc., USA) and the AxoScope software (Axon) were used for data capture and analysis. In order to measure conduction velocity of a given CAP wave, the distance between the stimulating and recording electrodes was divided by the time elapsed between the initiation of the stimulus and the time when the positive peak of that CAP wave was reached. Strength-duration curves using constant-voltage square waves were used to determine rheobase and chronaxy (12). Rheobase was measured as the threshold stimulus voltage for an active response with a long-duration pulse $(1000 \mu \mathrm{s})$ and chronaxy as the pulse-width corresponding to twice the rheobase.

Nerves were first stimulated at $0.2 \mathrm{~Hz}$ for $120 \mathrm{~min}$, a time period sufficient to achieve stable recording. Subsequently, CAP parameters were determined. Nerves that showed no change in CAP amplitude during the last $30 \mathrm{~min}$ were included in the study. Subsequently, we recorded CAPs evoked at 20,50, and $100 \mathrm{~Hz}$. Each stimulation period lasted $2 \mathrm{~min}$, with 5 -min intervals of $0.2 \mathrm{~Hz}$ stimulation.

\section{Preparation for transmission electron microscopy}

Anesthetized animals $(n=6)$ were perfused with $4 \%$ paraformaldehyde. After perfusion, the distal SN was dissected and fixed in Karnovsky solution (2\% paraformaldehyde, $2.5 \%$ glutaraldehyde in $0.1 \mathrm{M}$ sodium cacodylate buffer, $\mathrm{pH}$ 7.2) for $3 \mathrm{~h}$, at room temperature. Post-fixation was done with $1 \%$ osmium tetroxide, $0.8 \%$ potassium ferricyanide, and $5 \mathrm{mM}$ calcium chloride for $1 \mathrm{~h}$ in the dark. The contrast staining was done en bloc with uranyl acetate and the tissue was dehydrated in a series of increasing acetone concentrations. After dehydration, the tissue was embedded in Spurr resin. Ultra-thin sections $(70 \mathrm{~nm})$ were cut with an ultramicrotome and examined using a Jeol 1011 transmission electron microscope (Jeol, Japan). The images collected were analyzed with the Image $\mathrm{J}$ software (National Institutes of Health, USA) and grouped into three frequency distributions of axonal diameter, 1.00-4.99, 5.00-7.99, and 8.00$11.99 \mu \mathrm{m}$.

\section{Statistical analysis}

Results are reported as means $\pm S E$. Data were analyzed using the Student $t$-test or ANOVA followed by a contrast test or a non-parametric test, as appropriate. Results were considered to be significant at $\mathrm{P} \leq 0.05$. 


\section{Results}

\section{Electrophysiological alterations}

The 14-day immobilization of the right hind leg affected body weight and the mass of the right soleus and gastrocnemius muscles. The mean body weights of the control and immobilized groups were $370.3 \pm 7.20$ and $297.3 \pm 14.55 \mathrm{~g}$, respectively. The soleus of the control and immobilized groups weighed $0.18 \pm 0.004$ and $0.11 \pm 0.006 \mathrm{~g}$, and the gastrocnemius weighed $2.24 \pm 0.133$ and $1.60 \pm 0.100 \mathrm{~g}$, respectively. All three differences were statistically significant $(P<0.05$, unpaired Student $t$-test, $\mathrm{n}=15$ ).

Immobilization altered the CAP (Figure $1 \mathrm{~A}$ and $\mathrm{B}$ ). It significantly increased nerve chronaxy (52.31 \pm 1.95 to $59.71 \pm 2.79 \mu \mathrm{s}$; Figure $1 \mathrm{C}$ ) and decreased rheobase $(3.13 \pm 0.05$ to $2.84 \pm 0.06 \mathrm{~V}$; Figure 1D) compared to the control values $(P<0.05$, for each group, unpaired Student $t$-test, $\mathrm{n}=15$ ). These altered electrophysiological parameters indicate that immobilization induced changes in nerve excitability.

Since excitability was altered by immobilization, we examined whether immobilization affected the CAP waveform. Typically, under our stimulation and recording conditions, CAPs consisted of two distinct components or waves (Figure $1 \mathrm{~A}$ and $\mathrm{B}$ ). $\mathrm{A}$ third component wave is frequently observed at the end of the stabilization period, but it often progressively diminishes in amplitude, becoming indistinguishable from baseline noise during this period (13). In the present study, we analyzed only the first two waves of the CAP.

We analyzed the effect of immobilization on CAP conduction velocities and amplitudes in preparations stimulated at a baseline frequency and at higher frequencies. Immobilization significantly decreased the conduction velocity of the first wave (Figure 1E, $\mathrm{P}<0.05$, ANOVA) but had no significant effect on the conduction velocity of the second wave (Figure 1F, ANOVA). The amplitudes of the CAP waves were differentially affected by immobilization. The first component was significantly decreased (Figure 1G) while the amplitude of the second component was significantly enhanced (Figure $1 \mathrm{H}$; both $\mathrm{P}<0.05$, ANOVA).

The amplitudes and the conduction velocities of the first and second CAP waves were also analyzed at various frequencies $(0.2-100 \mathrm{~Hz})$. Increasing frequency tended to decrease CAP conduction velocity and amplitude in both control and immobilized animals (Figure 1E-H) but these effects did not reach statistical significance (ANOVA).

The magnitude of the decreases in amplitude and velocity of the first and second CAP components with increasing frequency $(0.2-100 \mathrm{~Hz})$ in control and in immobilized animals was also analyzed and the values are reported in Table 1. The frequency-dependent decreases were larger for CAP amplitude than for conduction velocity ( $95 \%$ confidence interval), independent of the condition. Comparing the size of the decrease of a given parameter for different conditions, the decrease in amplitude and conduction velocity for the first component were proportionately larger (without reaching statistical significance) in animals with an immobilized limb than in controls (Table 1). With respect to the second CAP component, the alteration of conduction velocity with increase in frequency was proportionally the same in control and in immobilized animals; for the alterations in amplitude, the decrease in amplitude with increase in frequency was smaller in immobilized animals than in controls (Table 1).

\section{Morphological alterations}

Electron microscopy revealed axonal degeneration of $\mathrm{SN}$ from immobilized limbs. The morphological changes were predominantly a fragmentation of the myelin sheath and loss of integrity of the respective axon. This degeneration affected predominantly the axons with the largest diameter (Figure 2). In the same tissue samples, an increase in the number of small and medium size diameter axons was observed (Figure 3). In order to quantify this, the collected images were analyzed for the frequency distribution of SN axonal diameter. Three size intervals were selected: 1.00-4.99, 5.00-7.99, and 8.00$11.99 \mu \mathrm{m}$. In the control group, of 101 axons measured, $22.7,50.5$, and $26.7 \%$ of axons had diameters between 1.00-4.99, 5.00-7.99, and 8.00-11.99 $\mu \mathrm{m}$, respectively. Of 204 axons from the immobilized group, 38.7, 52.4, and $8.8 \%$ of axons had diameters between 1.00-4.99, 5.007.99 , and $8.00-11.99 \mu \mathrm{M}$, respectively. The frequency distributions in control and immobilized groups differed significantly $(P<0.001$, chi-square test). A large number of the small and medium size diameter axons showed a cluster organization suggestive of an on-going regenerative process (Figure 2).

\section{Discussion}

The major discovery of the present study is that immobilization causes alterations in excitability and morphological structure of a peripheral nerve of an immobilized limb. It has been reported that long-lasting immobilization causes a decrease in functional performance and atrophy of muscles of the immobilized limb $(4,5,10)$. To date no studies have examined morphological and functional changes in the nerves of immobilized muscles. Surprisingly, and contradictory to what might be expected based on studies with immobilized skeletal muscle, there was an increase in neuronal excitability in nerves from immobilized skeletal muscle (Figure 1).

No less surprising was the pattern of myelin fragmentation and axonal degeneration, affecting predominantly the axons with the largest diameters, and of regeneration, which was characterized by the appearance of clusters of axons of medium size diameter. To the best of our 


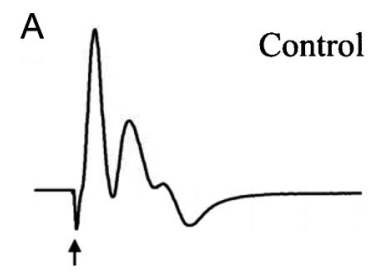

C

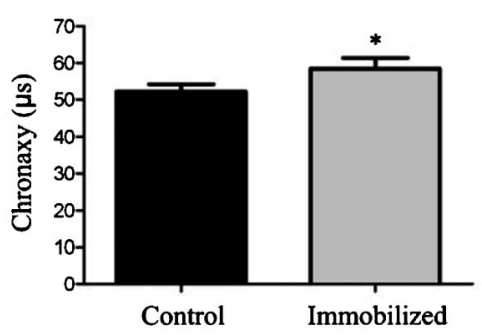

E

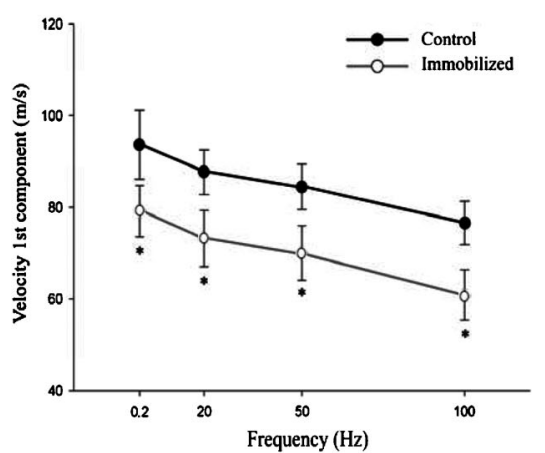

G

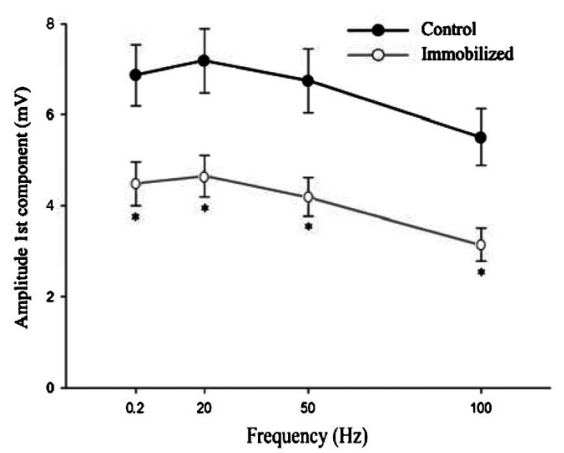

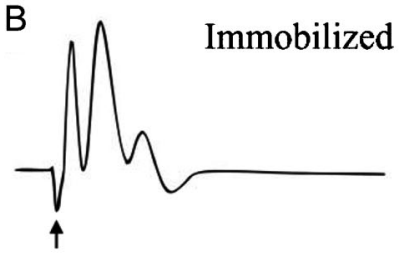

D

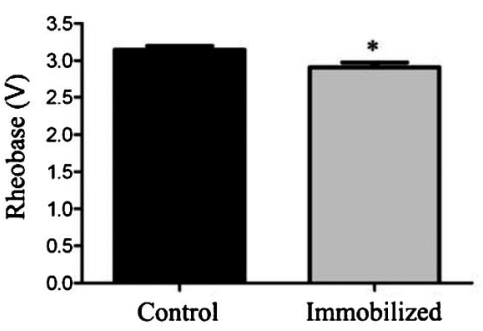

F

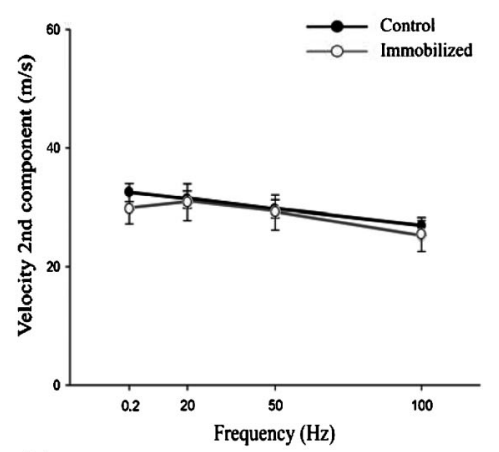

$\mathrm{H}$

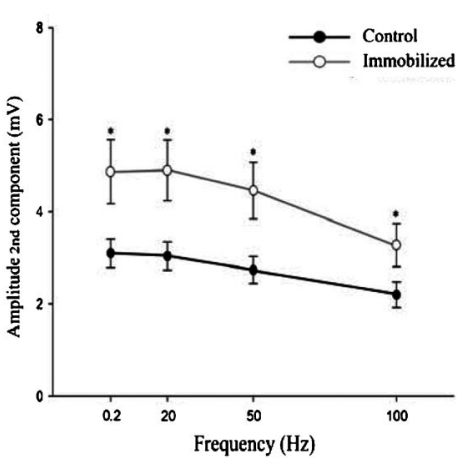

Figure 1. Effect of immobilization on compound action potential (CAP) parameters. $A$ and $B$ show representative CAP tracings in control $(A)$ and immobilized $(B)$ rats. $C$ and $D$ show chronaxy $(C)$ and rheobase $(D)$ in control and immobilized rats. $E, F, G$, and $H$ show conduction velocity of first $(E)$ and second $(F)$ components and positive amplitude of first $(G)$ and second $(H)$ components of CAP at different frequencies in control and immobilized rats. Data are reported as means $\pm S E(n=13$ and $n=15$ for control and immobilized rats, respectively). ${ }^{*} \mathrm{P}<0.05$ control vs immobilized ( $C$ and $D$, non-paired Student $t$-test; $E, F, G$ and $H$, at a given frequency, ANOVA followed by the Bonferroni post hoc test). 
Table 1. Comparison of amplitude and velocity of the waves of the compound action potential (CAP) at 0.2 and $100.0 \mathrm{~Hz}$ for control vs immobilized conditions.

\begin{tabular}{|c|c|c|c|c|c|c|c|}
\hline \multirow[t]{2}{*}{ Parameter } & \multicolumn{3}{|c|}{ Control group $(n=13)$} & \multicolumn{3}{|c|}{ Immobilized group $(n=15)$} & \multirow{2}{*}{$\begin{array}{l}\text { Difference } \\
\text { (ID100- } \\
\text { CD100) }\end{array}$} \\
\hline & $\begin{array}{c}\text { Mean } \pm \text { SE of } \\
\text { absolute value at } \\
0.2 \mathrm{~Hz}\end{array}$ & $\begin{array}{c}\text { Decrease } \\
\text { observed } \\
\text { at } 100 \mathrm{~Hz} \\
(\mathrm{CD} 100)\end{array}$ & $\begin{array}{l}95 \% \mathrm{Cl} \text { for } \\
\text { CD100 }\end{array}$ & $\begin{array}{c}\text { Mean } \pm \text { SE of } \\
\text { absolute value at } \\
0.2 \mathrm{~Hz}\end{array}$ & $\begin{array}{c}\text { Decrease } \\
\text { observed at } \\
100 \mathrm{~Hz} \\
\text { (ID100) }\end{array}$ & $\begin{array}{l}95 \% \mathrm{Cl} \text { for } \\
\text { ID100 }\end{array}$ & \\
\hline $\begin{array}{l}\text { Velocity 1st } \\
\text { component }\end{array}$ & $93.6 \pm 7.49(\mathrm{~m} / \mathrm{s})$ & $16.9 \%$ & $12.6-21.3$ & $79.1 \pm 5.59(\mathrm{~m} / \mathrm{s})$ & $22.08 \%$ & $13.62-30.55$ & 5.15 \\
\hline $\begin{array}{l}\text { Amplitude 1st } \\
\text { component }\end{array}$ & $7.0 \pm 0.49(\mathrm{mV})$ & $28.8 \%$ & $23.4-34.2$ & $4.6 \pm 0.45(\mathrm{mV})$ & $35.84 \%$ & $30.94-40.73$ & 7.04 \\
\hline $\begin{array}{l}\text { Velocity 2nd } \\
\text { component }\end{array}$ & $32.5 \pm 1.55(\mathrm{~m} / \mathrm{s})$ & $17.3 \%$ & $14.6-20.0$ & $32.9 \pm 2.00(\mathrm{~m} / \mathrm{s})$ & $21.64 \%$ & $18.50-24.79$ & 4.35 \\
\hline $\begin{array}{l}\text { Amplitude 2nd } \\
\text { component }\end{array}$ & $3.3 \pm 0.30(\mathrm{mV})$ & $37.9 \%$ & $31.9-43.8$ & $4.9 \pm 0.69(\mathrm{mV})$ & $33.95 \%$ & 28.76-39.15 & -3.93 \\
\hline
\end{tabular}

aDifference: difference in decrease from immobilized to control observed at $100 \mathrm{~Hz}$. CD100: decrease, in control, upon stimulation at $100 \mathrm{~Hz}$ reported as \% of value at $0.2 \mathrm{~Hz}$; ID100: decrease, in immobilized condition, upon stimulation at $100 \mathrm{~Hz}$ reported as \% of value at $0.2 \mathrm{~Hz}$.

knowledge, these immobilization-induced functional and morphological changes in nerves within immobilized muscles have never been reported.

In order to diminish the scars and ulcerations of nonmobilized limbs, adaptations of the immobilization procedures have been developed $(4,5,10)$. The weights of the gastrocnemius and the soleus muscle were decreased in this study, confirming that our method promoted the expected negative trophic effects on muscles. We also measured body weight, which was decreased (data not shown), and these data also agree with the literature $(4,5)$. These results support the contention that the nerve alterations observed were really obtained under appropriate conditions of limb immobilization.

Concerning the decrease in rheobase value, which reflects an increase in excitability, it may result from alterations in several factors related to active or passive properties of the axons. It may result from leakage conductivity and membrane time constant, geometric

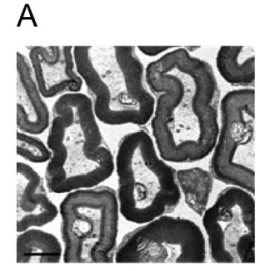

C

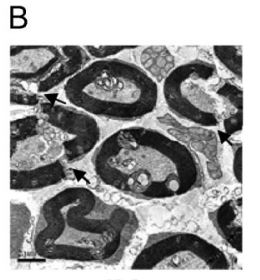

IM

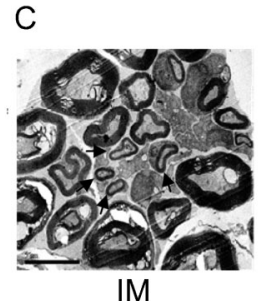

IM
Figure 2. Electron micrographs of sciatic nerves (SN) from control $(A)$ and immobilized rats $(B$ and $C)$. Note the immobilization-induced degeneration of myelin sheath of large diameter axons (arrows) of immobilized animals $(B)$. Clusters of thin fibers (arrows) can also be seen in the $\mathrm{SN}$ of immobilized animals $(C)$. Magnification bars: 2, 2, and $5 \mu \mathrm{m}$ for $A, B$, and $C$, respectively. C: control; IM: immobilized. factors related to the extracellular medium or voltagedependent alterations in sodium conductance (12). Geometric factors are believed not to play a role, since all procedures related to the nerve condition in the chamber during in vitro evaluation were the same for control and experimental animals.

Little can be suggested about the contribution of the other factors to the observed increase in excitability. One aspect of this decrease in rheobase, however, deserves consideration: that it occurred simultaneous with an increase in chronaxy. If this rheobase diminution was caused only by primary alteration of active factors related to inward $\mathrm{Na}^{+}$current and without membrane time constant modification, then a decrease in voltage threshold for action potential firing would imply alterations in rheobase and chronaxy in the same direction, i.e., decreases in both values. This did not occur, suggesting that an increase in membrane time constant occurred. It is the product of membrane resistance and membrane capacitance, which determines the membrane time constant $(14,15)$. Therefore, one or both of these parameters is likely to be altered in immobilization. It is

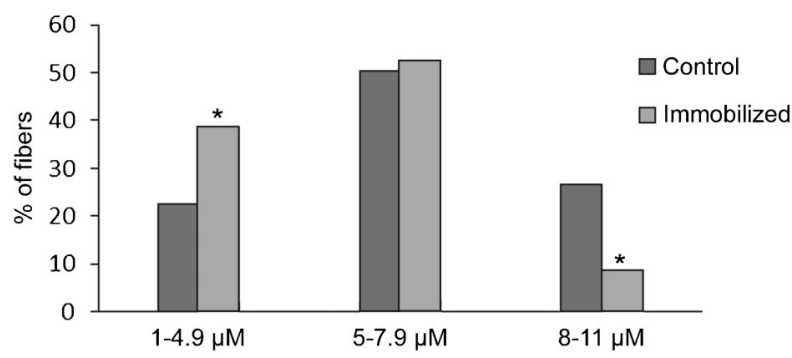

Figure 3. Frequency distributions of sciatic nerve fiber diameters in 101 fibers from control animals and 204 fibers from experimental animals. ${ }^{*} \mathrm{P}<0.001$, compared to control (chi-square test). 
known that axonal myelination decreases membrane capacitance (15). Electron microscopy data showed that myelin fragmentation occurred. Since fragmentation is a progressive alteration, it is likely that, in some axons, alteration of myelin sheath occurred before full fragmentation, diminishing membrane capacitance. This supports the hypothesis that the increase in membrane chronaxy occurred through a primary increase in membrane capacitance.

Immobilization had a depressive effect on both the amplitude and the conduction velocity of the first CAP component, yet it caused an increase in amplitude of the second component but did not affect conduction velocity. It is difficult to deduce the cause of modifications of the first and second components from the available data. This is because modifications in CAP component waves may result from changes at the level of individual axons, like the $\mathrm{dV} / \mathrm{dt}$ of intracellular axonal action potentials, or from population effects, like the synchronization of intracellular axonal action potentials on the nerve $(16,17)$.

It is possible that immobilization acted via different mechanisms in the two components of the CAP, so as to promote nearly opposite effects. This hypothesis was supported by electron microscopy data. Axonal degeneration, probably related to myelin fragmentation, affected predominantly the axons with the largest diameter, which may explain the decrease in velocity and amplitude of the first wave of action potential. The increase in the number of small and medium size diameter axons partially due to cluster organization suggestive of the regenerative process (Figure 2B and $\mathrm{C}$ ) could explain the increase in amplitude of the second component without alteration of conduction velocity. Based on the conduction velocity and on classifications of mammalian nerve fibers (18), for the two CAP components of rat SN, we suggest that the first consists of contributions of $A \alpha$ fibers (50-120 m/s) and the second consists of $A \beta$ and $A \gamma$ fibers (15-70 m/s) (19-21). This would lead to the conclusion that, regarding myelinated fibers, immobilization affects predominantly A $\alpha$ fibers.

We cannot exclude the possibility that an alternative mechanism might have contributed to the increase in the second component amplitude. The contribution of an

\section{References}

1. Raso VV, Barbieri $\mathrm{CH}$, Mazzer N, Fasan VS. Can therapeutic ultrasound influence the regeneration of peripheral nerves? J Neurosci Methods 2005; 142: 185-192, doi: 10.1016/j.jneumeth.2004.08.016.

2. Higashimori $H$, Whetzel TP, Mahmood T, Carlsen RC. Peripheral axon caliber and conduction velocity are decreased after burn injury in mice. Muscle Nerve 2005; 31: 610-620, doi: 10.1002/mus.20306.

3. Rosenkranz K, Kacar A, Rothwell JC. Differential modulation of motor cortical plasticity and excitability in early and late action potential of a given axon to any component depends on its conduction velocity. Therefore, with a proportionally uniform decrease in conduction velocity for all axons, only the first component loses contribution. The second component, however, while likely also losing contribution from the slowest axons, probably accumulates the contributions of the axons that previously contributed to the first component.

It is documented that stimulation at frequencies $\geqslant 50 \mathrm{~Hz}$ for several minutes causes a decrease in action potential amplitude and conduction velocity. This depressive effect has been attributed to reversible microalterations of the structure of the node of Ranvier (22). Since we observed degeneration with electron microscopy, we investigated whether the depressive effect on CAP parameters by stimulation at high frequency was amplified. Our data, in accordance with the literature, showed a depressive effect on CAP. The depressive effect on conduction velocity seemed to be more pronounced with immobilization, since at $100 \mathrm{~Hz}$ the velocity of the first and second CAP components declined more with immobilization than in controls (Table 1). With respect to conduction velocity, however, the size of the decrease at $100 \mathrm{~Hz}$ was larger for the first and smaller for the second component. We have no explanation for this observation so far.

In conclusion, we have demonstrated that long-lasting immobilization has a depressive effect on nerve function. Our data raise several questions with clinical implications. For example, could the primary nerve degeneration be decreased or avoided by nerve stimulation during immobilization? This study shows that not only muscles but, at least in rats, nerve function also deteriorates with long-lasting immobilization. This brings the question whether the same happens in humans and, if it does, how to properly stimulate the nerve and not only the muscles to achieve good nerve preservation.

\section{Acknowledgments}

We are thankful to Dr. Daniel Weinreich for the orthographic revision. Research supported by CNPq, CAPES and FUNCAP. phases of human motor learning. J Neurosci 2007; 27: 12058-12066, doi: 10.1523/JNEUROSCI.2663-07.2007.

4. Noyes FR. Functional properties of knee ligaments and alterations induced by immobilization: a correlative biomechanical and histological study in primates. Clin Orthop Relat Res 1977; 123: 210-242.

5. Appell HJ. Muscular atrophy following immobilisation. A review. Sports Med 1990; 10: 42-58, doi: 10.2165/ 00007256-199010010-00005.

6. Ishido M, Kami K, Masuhara M. In vivo expression patterns 
of MyoD, p21, and $\mathrm{Rb}$ proteins in myonuclei and satellite cells of denervated rat skeletal muscle. Am J Physiol Cell Physiol 2004; 287: C484-C493, doi: 10.1152/ajpcell.00080.2004.

7. Kostrominova TY, Dow DE, Dennis RG, Miller RA, Faulkner JA. Comparison of gene expression of 2-mo denervated, 2mo stimulated-denervated, and control rat skeletal muscles. Physiol Genomics 2005; 22: 227-243, doi: 10.1152/physiolgenomics.00210.2004.

8. Billington L, Carlson BM. The recovery of long-term denervated rat muscles after Marcaine treatment and grafting. J Neurol Sci 1996; 144: 147-155, doi: 10.1016/ S0022-510X(96)00219-5.

9. Dow DE, Cederna PS, Hassett CA, Kostrominova TY, Faulkner JA, Dennis RG. Number of contractions to maintain mass and force of a denervated rat muscle. Muscle Nerve 2004; 30: 77-86, doi: 10.1002/mus.20054.

10. Santos-Júnior FFU, Alves JSM, Machado ANN, Carlos PS, Ferraz ASM, Barbosa R, et al. Morphometric alterations in respiratory muscle of rats submitted to paw immobilization. Rev Bras Med Esporte 2010; 16: 215-218, doi: 10.1590/ S1517-86922010000300012.

11. Lima-Accioly PM, Lavor-Porto PR, Cavalcante FS, Magalhaes PJ, Lahlou S, Morais SM, et al. Essential oil of croton nepetaefolius and its main constituent, 1,8-cineole, block excitability of rat sciatic nerve in vitro. Clin Exp Pharmacol Physiol 2006; 33: 1158-1163, doi: 10.1111/ j.1440-1681.2006.04494.x.

12. Holsheimer J, Dijkstra EA, Demeulemeester H, Nuttin B. Chronaxy calculated from current-duration and voltageduration data. J Neurosci Methods 2000; 97: 45-50, doi: 10.1016/S0165-0270(00)00163-1.

13. Leal-Cardoso JH, da Silva-Alves KS, Ferreira-da-Silva FW, dos Santos-Nascimento T, Joca HC, de Macedo $\mathrm{FH}$, et al. Linalool blocks excitability in peripheral nerves and voltagedependent $\mathrm{Na}^{+}$current in dissociated dorsal root ganglia neurons. Eur J Pharmacol 2010; 645: 86-93, doi: 10.1016/ j.ejphar.2010.07.014.

14. Holsheimer J, Demeulemeester H, Nuttin B, de Sutter P. Identification of the target neuronal elements in electrical deep brain stimulation. Eur J Neurosci 2000; 12: 4573-4577.

15. Ashley Z, Sutherland H, Lanmuller H, Unger E, Li F, Mayr $W$, et al. Determination of the chronaxy and rheobase of denervated limb muscles in conscious rabbits. Artif Organs 2005; 29: 212-215, doi: 10.1111/j.1525-1594.2005.29037.x.

16. Brismar $\mathrm{T}$. Electrical properties of isolated demyelinated rat nerve fibres. Acta Physiol Scand 1981; 113: 161-166, doi: 10.1111/j.1748-1716.1981.tb06877.x.

17. Chang MH, Liu LH, Lee YC, Hsieh PF. Alteration of proximal conduction velocity at distal nerve injury in carpal tunnel syndrome: demyelinating versus axonal change. J Clin Neurophysiol 2008; 25: 161-166, doi: 10.1097/ WNP.0b013e3181775981.

18. Aidley DJ. The physiology of excitable cells. 4th edn. Cambridge: Cambridge University Press; 1998.

19. Hayami T, Iramina K, Chen X, Sunagawa K. Simulation study on the effect of fiber loss to the compound action potential of a sural nerve. Conf Proc IEEE Eng Med Biol Soc 2007; 2007: 2396-2399.

20. Erlanger J, Gasser HS. Electrical signs of nervous activity. Am J Psychiatry 1937; 93: 1472-1473.

21. Catterall W, Mackie K. Local anesthesic. In: Goodman NLS, Gilman A (Editors), The pharmacological basis of therapeutics. 9th edn. New York: McGraw-Hill Companies; 1996. p 331-334.

22. Junge D. Nerve and muscle excitation. Massachusetts: Sinauer Associates; 1976. 